\title{
Glycosaminoglycans from Renal Brush Border Membranes of Rabbits
}

\author{
Hiroshi Munakata, Mamoru Isemura, Junichiro \\ Aikawa, Chie Kodama and Zensaku Yosizawa \\ Department of Biochemistry, Tohoku University School of \\ Medicine, Sendai 980
}

\begin{abstract}
Munakata, H., Isemura, M., Aikawa, J., Kodama, C. and Yosizawa, Z. Glycosaminoglycans from Renal Brush Border Membranes of Rabbits. Tohoku J. exp. Med., 1985, 145 (4), 353-358 — Brush border membranes were prepared from the rabbit kidney cortex by sucrose density gradient centrifugation and digested with pronase. A glycosaminoglycan fraction was isolated from the digest by ion-exchange chromatography. Electrophoretogram on cellulose acetate membrane (Separax) of the glycosaminoglycan fraction indicated the presence of two components, which were identified as chondroitin sulfate $\mathrm{A} / \mathrm{C}$ and dermatan sulfate on the basis of their mobility in Separax electrophoresis and sensitivity to chondroitinase ABC. Neither hyaluronic acid nor heparan sulfate was detected. The results of high-performance liquid chromatography of the pyridylamino derivatives of unsaturated disaccharides in the digests with chondroitinase $\mathrm{AC}$ and $\mathrm{ABC}$ of the glycosaminoglycan fraction indicated that the ratio of chondroitin 4 -sulfate, chondroitin 6 -sulfate and dermatan sulfate was $5.5: 1.0: 9.1$ glycosaminoglycans; brush border membrane; kidney
\end{abstract}

The brush border membrane is located at cell apex exposed to the lumen of the proximal convoluted tubule, and its function as transportation of various substances in a resorption process has been extensively studied (Aronson and Sacktor 1975).

Glycosaminoglycans (GAGs) are polyanionic and hydrophilic substances, and one possible function is to regulate the exchange of water and electrolyte (Murata 1982). GAGs in the kidney have been extensively investigated and it is now established that the mammalian kidney contains heparan sulfate, hyaluronic acid, dermatan sulfate, chondroitin sulfate and oversulfated dermatan sulfate in decreasing amounts in this order, although there may be qualitative and quantita-

Received August 24, 1984; accepted for publication September 26, 1984.

Abbreviations : GAG, glycosaminoglycan ; HPLC, high-performance liquid chromatography ; $\Delta \mathrm{Di}$-0S, $\quad 2$-acetamido-2-deoxy-3-O-(4-deoxy- $\alpha$-L-threo-hex-4-enepyranosyluronic acid)-D-galactose ; $\Delta$ Di-4S, 2-acetamido-2-deoxy-3-O-(4-deoxy- $\alpha$-L-threo-hex-4-enepyranosyluronic acid)-4-O-sulfo-D-galactose ; $\Delta$ Di-6S, $\quad 2$-acetamido-2-denxy-3-O-(4-deoxy- $\alpha$-Lthreo-hex-4-enepyranosyluronic acid)-6-O-sulfo-D-galactose, $\Delta$ Di-diS, 2-acetamido-2-deoxy-3O-(4-deoxy-2- or 3-O-sulfo- $\alpha$-L-threo-hex-4-enepyranosyluronic acid)-4- or 6-O-sulfo-D-galactose. 
tive differences among species (Murata 1982). There has been, however, little information concerning the distribution of individual GAG in specific renal structural components, although proteoheparan sulfate and heparan sulfate have been isolated from the glomerular basement membrane (Kanwar and Farquhar 1979 ; Stow et al. 1983).

In the present work, we examined the GAG composition of the renal brush border membrane.

\section{Materials and Methods}

Materials. Pronase was obtained from Kaken Kagaku Co., Tokyo. Chondroitinase $\mathrm{AC}$ and chondroitinase $\mathrm{ABC}$ were purchased from Seikagaku Kogyo Co., Tokyo. Other materials were those described previously (Munakata and Yosizawa 1980a, b).

Preparation of brush border membranes. Rabbit renal brush border membranes were isolated by the method of Aronson and Sacktor (1975). Kidneys from eight rabbits were quickly removed from animals, and were chilled in cold 0.5M sucrose, decapsulated, and defatted. All subsequent steps in the procedure were carried out at $4^{\circ} \mathrm{C}$. The cortices were dissected, weighed, and then finely sliced. The tissue was homogenized by hand in $0.5 \mathrm{M}$ sucrose with a Dounce homogenizer, with a ratio of $1 \mathrm{~g}$ of tissue to $6 \mathrm{ml}$ of $0.5 \mathrm{M}$ sucrose. The homogenate was centrifuged rapidly and briefly by setting the centrifuge to attain $4,500 \times \mathrm{g}$ and when this force was reached stopping the centrifuge immediately. The supernatant was saved. The residue was resuspended in one-half of original volume of $0.5 \mathrm{M}$ sucrose, homogenized with three strokes of the Potter-Elvehjem pestle, and recentrifuged in a same manner. The combined supernatant was fractionated by layering the suspension on a discontinuous gradient comprising $8 \mathrm{ml}$ each of 1.7 and $1.4 \mathrm{M}$ sucrose. The tubes were centrifuged at $24,000 \mathrm{rpm}$ for $60 \mathrm{~min}$ using RPS-30 rotor in a Hitachi RP-60 centrifuge. The pink fluffy layer containing the brush border membranes was distributed at the interface of the $1.4 \mathrm{M}$ and $0.5 \mathrm{M}$ sucrose zone. This layer plus the lower halves of the overlying supernatant was aspirated and resuspended in $0.5 \mathrm{M}$ sucrose to make the original volume. The suspension was centrifuged at $4,300 \times \mathrm{g}$ for $30 \mathrm{~min}$. The loose pink sediments, together with the supernatant, were separated from the pellets. The mixture was centrifuged at $31,000 \times \mathrm{g}$ for $5 \mathrm{~min}$. The resulting supernatant was discarded.

The brush border membranes were visible as the loosely packed pink portion of the pellet overlying a dense, tightly packed brownish colored core containing primarily mitochondria. The brush border membranes were washed out from the pellet, resuspended in the sucrose, and centrifuged again at $31,000 \times \mathrm{g}$ for $5 \mathrm{~min}$. The resulting pellet was resuspended in the sucrose and centrifuged for $10 \mathrm{~min}$ sequentially at 7,700,4,300, and $3,000 \times$ g. After each centrifugation, the supernatant was discarded and loosely packed brush border membranes were washed out from the diminishing densely packed contaminating portion of the pellet. The membranes were then suspended in $40 \mathrm{ml}$ of water and centrifuged at $31,000 \times \mathrm{g}$ for $30 \mathrm{~min}$. The pellet thus obtained was used as a brush border membrane fraction.

Isolation of GAGs. The membranes (350 $\mathrm{mg}$, wet weight) were suspended in $1.2 \mathrm{ml}$ of water. To the suspension were added $3.2 \mathrm{ml}$ of methanol and $1.6 \mathrm{ml}$ of chloroform with stirring. The mixture was left at room temperature for $1 \mathrm{hr}$ and centrifuged at 5,000 rpm for $30 \mathrm{~min}$. The pellet was washed with a mixture of chloroform-methanol-water $(3: 8: 4$, by volume) and dried in vacuo. The dried powder $(39 \mathrm{mg})$ was suspended in $2 \mathrm{ml}$ of $0.1 \mathrm{M}$ Tris-HCl buffer ( $\mathrm{pH} 7.8$ ) containing $0.01 \mathrm{M}$ calcium acetate and digested with $0.4 \mathrm{mg}$ of pronase at $45^{\circ} \mathrm{C}$ for $24 \mathrm{hr}$. Then, a half amount of pronase was added to the incubation mixture and digestion was continued for further $24 \mathrm{hr}$. To the chilled mixture was added dropwise chilled $50 \%$ trichloroacetic acid with stirring to give a final concentration of $7 \%$. 
The resulting precipitate was removed by centrifugation and the supernatant was dialyzed against water, and lyophilized. The lyophilized material was dissolved in $2 \mathrm{ml}$ of water and applied to a column $(0.8 \times 11 \mathrm{~cm})$ of DEAE-Sephadex A-25 $\left(\mathrm{Cl}^{-}\right.$form). The column was washed with $20 \mathrm{ml}$ each of water and $0.3 \mathrm{M} \mathrm{NaCl}$. These fractions did not contain uronic acid and were not studied further. Subsequently, elution was performed with $20 \mathrm{ml}$ of $2 \mathrm{M} \mathrm{NaCl}$. The eluate was dialyzed exhaustively against water and lyophilized, yielding the GAG fraction.

Electrophoresis on cellulose acetate membrane. Electrophoresis was carried out on cellulose acetate membrane (Separax) in pyridine-formate buffer $(\mathrm{pH} \mathrm{3.0)}$ ) at $1 \mathrm{~mA} / \mathrm{cm}$ for 25 min, and GAGs were located by staining with alcian blue as described previously (Munakata et al. 1980a).

HPLC analysis. The GAG fraction (400 ng as uronic acid) was digested with 0.1 unit of chondroitinase $\mathrm{AC}$ or 0.1 unit of chondroitinase $\mathrm{ABC}$ in $0.05 \mathrm{M}$ Tris-HCl buffer $(\mathrm{pH} 8.0)$ at $37^{\circ} \mathrm{C}$ for $3 \mathrm{hr}$. The unsaturated disaccharides released were subjected to reductive amination and the resulting pyridylamino derivatives were analyzed by HPLC on a column $(3.9 \times 300 \mathrm{~mm})$ of $\mu$ Bondapak C18 using Water's Liquid Chromatograph as described previously (Kodama et al. 1984). The individual GAG composition was calculated by the method described previously (Kodama et al. 1984).

Other methods. Uronic acid was measured by the modified carbazole-sulfuric acid method (Bitter and Muir 1962). Hexosamine was determined by the Elson-Morgan method after hydrolysis with $4 \mathrm{~N} \mathrm{HCl}$ at $100^{\circ} \mathrm{C}$ for $8 \mathrm{hr}$ (Boas, 1953).

\section{Results}

\section{Identification of GAGs}

From sixteen kidneys, $350 \mathrm{mg}$ (wet weight) of brush border membranes were obtained. The membranes were then delipidated and digested with pronase. DEAE-Sephadex A-25 column chromatography of the digest yielded $1 \mathrm{mg}$ of the glycosaminoglycan fraction. Chemical analysis indicated that the contents of uronic acid and hexosamine in the fraction were 32 and $37 \%$, respectively. Electrophoretogram on Separax indicated that the GAG fraction contained two alcian blue-stainable bands corresponding to those of standard chondroitin sulfate $\mathrm{A} / \mathrm{C}$ and dermatan sulfate (Fig. 1). After digestion of the GAG fraction with chondroitinase $\mathrm{ABC}$, no band was detected (data not shown). These results clearly indicate that the GAG fraction obtained from the renal brush border membranes contains chondroitin sulfate $\mathrm{A} / \mathrm{C}$ and dermatan sulfate. Neither hyaluronic acid nor heparan sulfate was detected.

\section{Composition of $G A G$}

The GAG fraction was divided into two portions. One portion was digested with chondroitinase $\mathrm{AC}$, and the other with chondroitinase $\mathrm{ABC}$. Each digest was subjected to reductive amination with 2-aminopyridine and sodium cyanoborohydride. The resulting pyridylamino derivatives of unsaturated disaccharide were analyzed by HPLC. The chromatograms are shown in Fig. 2. The composition of unsaturated disaccharides is summarized in Table I. The ratio of chondroitin 4-sulfate, chondroitin 6-sulfate and dermatan sulfate was calculated to be $5.5: 1.0: 9.1$ from these data. 


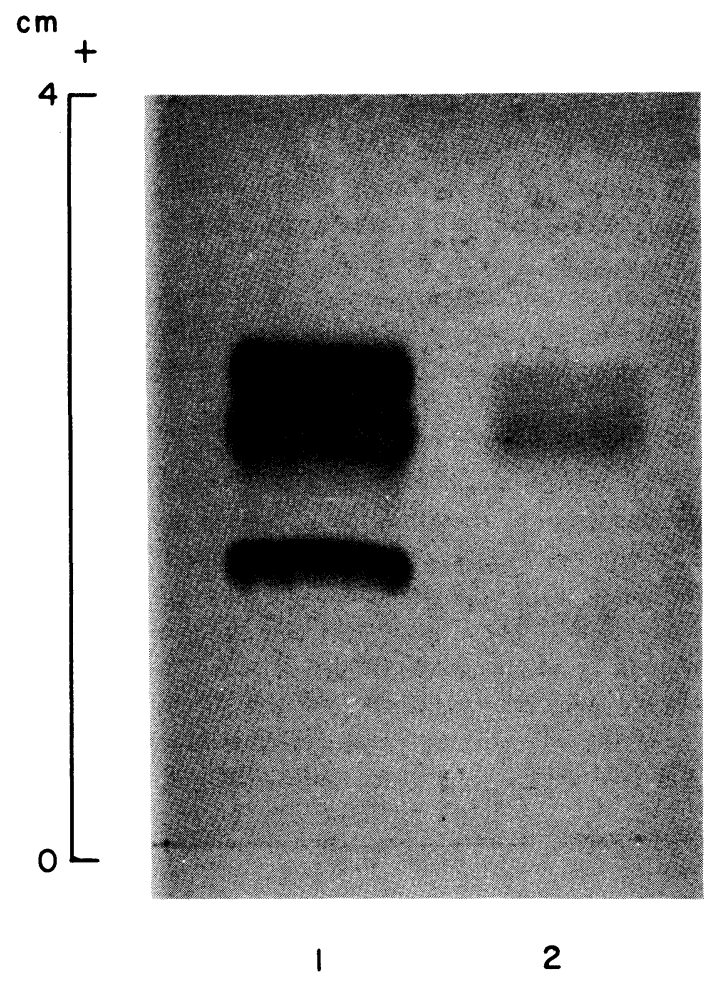

Fig. 1. Electrophoretogram of the GAG fraction obtained from the renal brush border membranes on cellulose acetate membrane (Separax). Electrophoresis was carried out in pyridine-formate buffer ( $\mathrm{pH} \mathrm{3.0)}$ at $1 \mathrm{~mA} / \mathrm{cm}$ for $25 \mathrm{~min}$. GAGs were located by staining with alcian blue $(0.1 \%$ in $1 \%$ acetic acid). 1, a mixture of chondroitin sulfate A, dermatan sulfate and hyaluronic acid (from top to bottom); 2, GAG fraction.

TABLE 1. Composition* of unsaturated disaccharides produced from the GAG fraction by digestion with chondroitinases

\begin{tabular}{ccccc}
\hline Enzyme & $\Delta$ Di-diS & $\Delta$ Di-4S & $\Delta$ Di-6S & $\Delta$ Di-0S \\
\hline Chondroitinase AC & 0 & 67 & 365 & 0 \\
Chondroitinase ABC & 9 & 84 & 1297 & 0 \\
\hline
\end{tabular}

* Expressed as pmol per GAG fraction (400 ng in terms of uronic acid).

\section{Discussion}

Although an isolation method of the renal brush border membrane has been developed (Berger and Sacktor 1970), there has been no information concerning GAGs in it. Therefore, we attempted to characterize GAGs in the brush border membrane. Since the yield of the GAG fraction was considerably low, quantitative determination of individual GAG was performed by a highly sensitive HPLC 


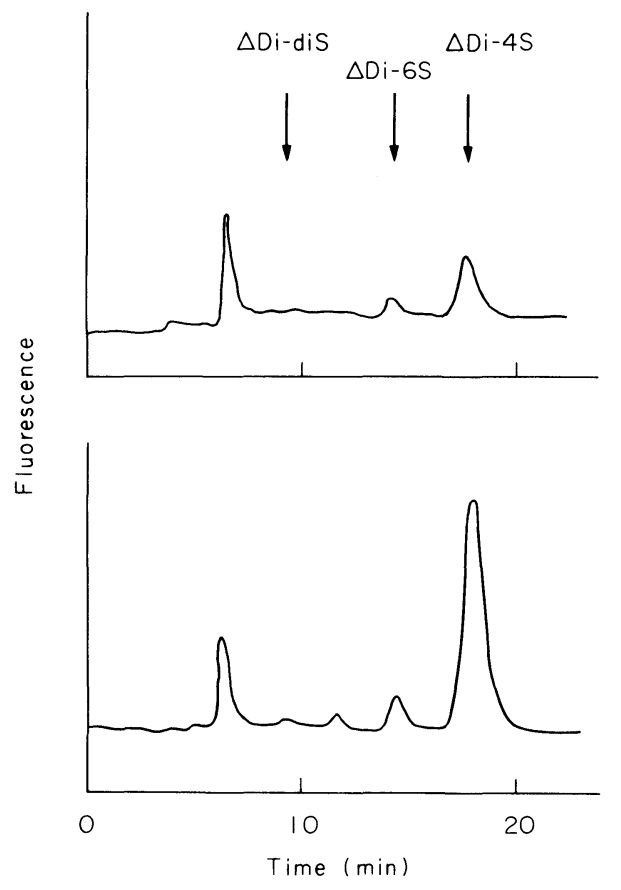

Fig. 2. HPLC profiles of the pyridylamino derivatives of unsaturated disaccharides from the GAG fraction. The GAG fraction (400 ng in terms of uronic acid) was digested with chondroitinase $\mathrm{AC}$ (upper) or chondroitinase $\mathrm{ABC}$ (lower). Resulting disaccharides were subjected to reductive amination and the resulting pyridylamino derivatives were analyzed by HPLC.

method developed recently by Kodama et al. (1984). The present data indicate that the renal brush border membrane contains chondroitin sulfate $\mathrm{A} / \mathrm{C}$ and dermatan sulfate, but that hyaluronic acid and heparan sulfate are absent.

Heparan sulfate has been shown to be the predominant GAG in kidneys (Murata 1983), and Kanwar and Farquhar (1979) reported that heparan sulfate is associated with the basement membrane.

In our previous study (Munakata and Yosizawa, 1980b), we isolated a sulfated glycoprotein from intestinal brush borders, histological appearance of which resembles that of the renal brush border. In the present study, we could not detect any sulfated glycopeptide in the pronase digest of the renal brush border membrane. There are two possible explanations for this observation: sulfated glycoprotein is absent in the renal brush border membrane; the membrane contains sulfated glycoprotein which does not yield non-dialyzable sulfated glycopeptide upon the pronase digestion. If the latter is the case, the sulfated glycoprotein in the renal brush border membrane must be different from typical one which is known to yield non-dialyzable glycopeptide by protease digestion (Yosizawa 1972). 


\section{Acknowledgments}

This work was supported in part by a Grant-in-Aid for Scientific Research from the Ministry of Education, Science and Culture of Japan.

\section{References}

1) Aronson, P.S. \& Sacktor, B. (1975) The $\mathrm{Na}^{+}$gradient-dependent transport of Dglucose in renal brush border membranes. $J$. biol. Chem., 250, 6032-6039.

2) Berger, S.J. \& Sacktor, B. (1970) Isolation and biochemical characterization of brush borders from rabbit kidney. J. Cell Biol., 47, 673-645.

3) Bitter, M. \& Muir, H. (1962) A modified uronic acid carbazole reaction. Analyt. Biochem., 4, 330-334.

4) Boas, N.F. (1953) Method for the determination of hexosamines in tissues. J. biol. Chem., 204, 553-563.

5) Kanwar, Y.S. \& Farquhar, M.G. (1979) Isolation of glycosaminoglycans (heparan sulfate) from glomerular basement membranes. Proc. nat. Acad. Sci., 76, 4493-4497.

6) Kodama, C., Ototani, N., Isemura, M. \& Yosizawa, Z. (1984) High performance liquid chromatography of pyridylamino derivatives of unsaturated disaccharides produced from chondroitin sulfate isomers by chondroitinases. J. Biochem., 96, 1283-1287.

7) Munakata, H. \& Yosizawa, Z. (1980a) Isolation and characterization of sulfated glycoprotein from a transplantable colorectal adenocarcinoma of rats. Biochim. biophys. Acta, 632, 412-417.

8) Munakata, H. \& Yosizawa, Z. (1980b) Isolation and characterization of sulfated glycoprotein from the brush border fraction and the soluble fraction of rabbit small intestine. J. Biochem., 87, 1559-1565.

9) Murata, K. (1982) Glycosaminoglycans of kindneys: normal and pathological aspects. In: Glycosaminoglycans and Proteoglycans in Physiological and Pathological Processes of Body System, edited by R.S. Varma \& R. Varma, Karger, Basel, pp. 135150.

10) Stow, J.L., Glasgow, E.F., Handley, C.J. \& Hascall, V.C. (1983) Biosynthesis of proteoglycans by isolated rabbit glomeruli. Arch. Biochem. Biophys., 225, 950-957.

11) Yosizawa, Z. (1972) Sulphated glycoproteins. In: Glycoproteins, edited by A. Gottschalk, 2nd ed., Elsevier, Amsterdam, pp. 1000-1018. 